"This is the accepted manuscript of the following article: U. Plaznik et al $2015 \mathrm{EPL}$ 111 57009, which has been published in final form at

https://iopscience.iop.org/article/10.1209/0295-5075/111/57009 “

\title{
Electrocaloric cooling: The importance of electric-energy recovery and heat regeneration
}

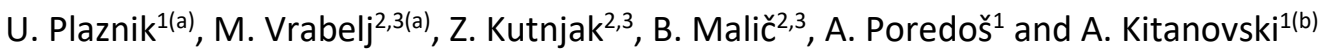

\author{
${ }^{1}$ University of Ljubljana, Faculty of Mechanical Engineering - Aškerčeva 6, 1000 Ljubljana, Slovenia \\ 2 Jožef Stefan Institute - Jamova cesta 39, 1000 Ljubljana, Slovenia \\ 3 Jožef Stefan International Postgraduate School - Jamova cesta 39, 1000 Ljubljana, Slovenia
}

PACS 77.70.+a - Pyroelectric and electrocaloric effects

PACS 88.05.-b - Energy analysis

\begin{abstract}
Here we explore the effect of electric-energy recovery and heat regeneration on the energy efficiency of an electrocaloric-cooling system. Furthermore, the influence of the polarization electric field hysteresis on the energy efficiency of the system is analysed. For the purposes of the analysis, the properties of $(1-\mathrm{x}) \mathrm{Pb}(\mathrm{Mg} 1 / 3 \mathrm{Nb} 2 / 3) \mathrm{O} 3-\mathrm{xPbTiO3}$ (PMN-100xPT) with $\mathrm{x}=0, \mathrm{x}=0.1$, and $\mathrm{x}=0.35$ are characterized. We show that if no heat is regenerated, even small irreversibilities in the electric circuit used to recover the electric energy can cause a significant drop in the achievable energy efficiency. On the other hand, when a heat regeneration process is considered and a realistic value for the degree of electric-energy recovery equal to $80 \%$ is assumed, the limit for the energy efficiency of a system employing PMN ceramics is estimated to be equal to $81 \%$ of the efficiency of a Carnot heat pump.
\end{abstract}

Introduction. - Electrocaloric cooling is being considered as an alternative to existing cooling technologies. In comparison with conventional vapour compression refrigeration it has the potential to provide refrigeration systems with minimal environmental impact, higher-energy efficiency and lower levels of noise and vibrations [1,2]. In this way, electrocaloric cooling is analogous to the more mature technology of magnetocaloric cooling [3]; however, in the case of electrocaloric-cooling systems there is no need of the expensive and heavy permanent magnets. Therefore, electrocaloric systems could be more compact and produced with lower costs. Furthermore, because of the strong insulating properties of some electrocaloric materials, and hence a negligible Joule heating, electrocaloric cooling could have an advantage over solid-state thermoelectric devices [4].

The idea of electrocaloric cooling is based on a physical phenomenon called the electrocaloric effect (ECE). The ECE is a property of polar materials whose dipolar entropy is changed when an electric field is applied [2,4-6]. If the electric field is increased under adiabatic conditions, the electrocaloric material (ECM) normally heats up. The opposite occurs when the electric field is reduced under adiabatic conditions, and so the ECM cools down. So far, a number of ECMs were discovered and characterized [7-10]. The values of the adiabatic temperature changes $\left(\Delta T_{E C}\right)$ usually range from 
a few $\mathrm{K}$ in bulk and thick-film ceramics [11-14] to a few tens of $\mathrm{K}$ in thin-film ceramics [15-18]. Similarly to the case of thin-film ceramics, the $\Delta T_{E C}$ in polymer films can reach up to a few tens of $K$ [19-21].

To use an ECM for the purposes of cooling and refrigeration, the ECM needs to be implemented in a working system. Different system concepts were proposed in the literature $[1,22-$ $27]$ and some of them have been realized as the first proof-of-concept devices $[25,28,29]$.

Recently, Moya et al. [10] analysed the work needed to drive the ECE in a variety of materials. Furthermore, they considered a certain degree of electric-energy recovery and showed that it can substantially reduce the total energy needed to drive the ECE. However, the influence of the heat regeneration and the electric-energy recovery on the energy efficiency of a cooling system in which an ECM is subjected to a thermodynamic cycle has yet to be analysed. In this letter our main focus is to investigate the influence of the electric-energy recovery and the heat regeneration on the coefficient of performance (COP) for an electrocaloric-cooling system employing (1 $\mathrm{x}) \mathrm{Pb}\left(\mathrm{Mg}_{1 / 3} \mathrm{Nb}_{2 / 3}\right) \mathrm{O}_{3}-\mathrm{xPbTiO} 3$ ( $\mathrm{PMN}-100 \mathrm{PPT}$ ) relaxor ferroelectric ceramics with $\mathrm{x}$ equal to $0,0.1$ and 0.35 .

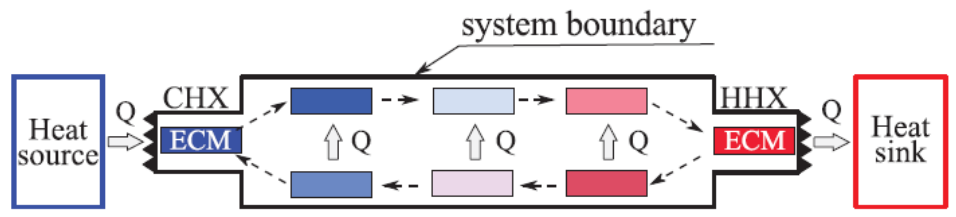

Fig. 1: (Colour on-line) Schematic presentation of a simple electrocaloric-cooling system with heat regeneration.

The thermodynamics of the process. - For the purposes of illustration, let us consider the system that is schematically presented in fig. 1. It consists of a heat exchanger adjacent to a heat source (CHX), a heat exchanger adjacent to a heat sink (HHX), and the ECM, which is moved from the heat source to the heat sink. It should be pointed out that for real applications more sophisticated design solutions will need to be used.

During the operation of the system heat is transferred between the system and its surroundings and the work is done on the system or performed by the system. We define the work done on the system as the electrical work done on the ECM, which can be written as

$$
w=\int E \times \mathrm{d} D,
$$

where $E$ is the electric field and $D$ the electric displacement field.

Normally, there are other effects that contribute to the work performed on an electrocaloriccooling system and also influence its cooling power, such as the energy needed to move the ECM (or to pump the heat transfer fluid), the energy dissipation due to viscous losses and friction, the irreversibilities due to heat transfer, etc. However, for the purposes of this analysis we will only focus on the electrical work done on the ECM and assume that the cooling energy of the system is equal to the cooling energy of the ECM.

Let us now focus on one element of the ECM, which is initially positioned in contact with the $H H X$. In the first process the electric field is changed from the starting field $E_{1}$ to the final field $E_{2}$ under adiabatic conditions, meaning that no heat is transferred from the $E C M$ to its surroundings during the first process. Due to the electric field change, the ECM is polarized and therefore it heats up. If the ECM possesses no polarization-electric field hysteresis, and other possible origins of internal entropy generation are neglected, no entropy is generated during the process. Therefore, the process is considered adiabatic and isentropic (fig. 2, process 1-2a). On the 
other hand, if the ECM possesses the polarization-electric field hysteresis, some entropy, denoted $\Delta \mathrm{s}_{\mathrm{H}}$, is generated (fig. 2, process 1-2).

In the second process the heat is first transferred from the ECM to the heat sink (fig. 2, process 2/2a-3) at a constant electric field E2 and the temperature of the ECM decreases. Next, the ECM leaves the HHX and is moved in the direction towards the CHX. On this path, the ECM exchanges heat with the pieces of ECM moving in the opposite direction (fig. 2, heat regeneration process 3-4). The piece of ECM under observation cools down during the process $2 / 2 a-4$. The polarization of ECMs is a function of temperature and will normally increase as the temperature of the ECM decreases, causing the electric field displacement to increase as well. Therefore, based on eq. (1), some work is performed on the system. Furthermore, since the ECM in the process $2 / 2 a-4$ is subjected to an electric field, a leakage current might be present, which increases the work performed during the process and generates undesired Joule heating.

In the third process (fig. 2, process 4-5/5a) the piece of ECM is in contact with the $\mathrm{CHX}$ and the electric field is reduced back to the starting value. The ECM is depolarized under adiabatic conditions and, due to the ECE, it cools down. In analogy with the second process, some entropy is generated in the case of the ECM with hysteresis, otherwise the process is isentropic.

In the last, fourth, process (fig. 2, process $5 / 5 a-6$ ), the heat is first transferred from the heat source to the ECM. Then the piece of ECM is moved in the direction towards the HHX and exchanges heat with the pieces of ECM moving in the opposite direction (fig. 2, heat regeneration process 6-1). In contrast to the second process, the ECM heats up during the fourth process and its polarization is decreased, leading to a decrease in the electric field displacement. The fourth process normally runs at zero electric field and therefore, despite of the change of the electric field displacement, no work is performed by the system according to eq. (1). Furthermore, the leakage current is not present.

(a)
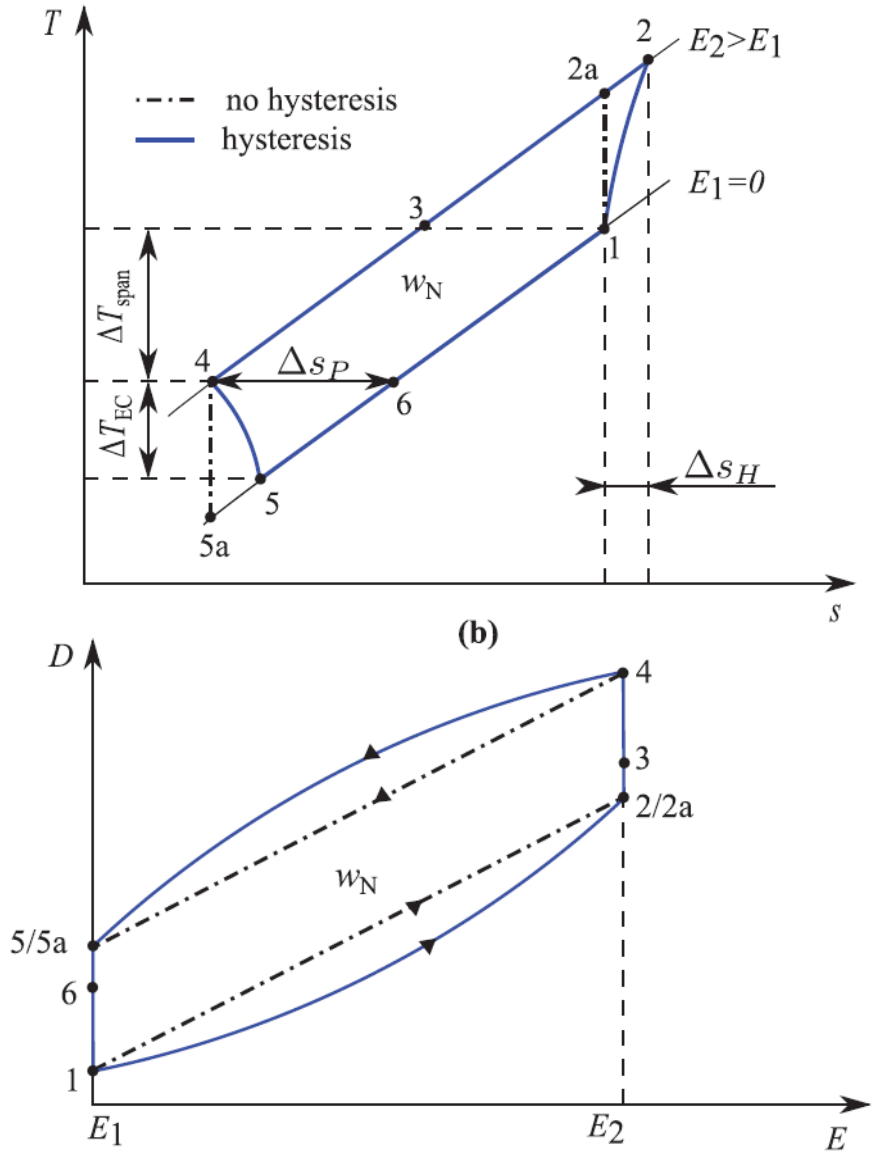

Fig. 2: (Colour on-line) Schematic presentation of the thermodynamic cycle to which the ECM is subjected. (a) Temperature-entropy diagram. (b) Electric displacement field-electric field diagram. 
The net work performed on the system equals the work performed on the ECM that was subjected to one thermodynamic cycle. In the case of negligible or very small leakage current (as in the case of the PMN-100xPT) the net work can be written as

$$
w_{\mathrm{N}}=\oint E \times \mathrm{d} D=w_{1-2 / 2 \mathrm{a}}+w_{2 \mathrm{a} / 2-4}+w_{4-5 / 5 \mathrm{a}} .
$$

Next, by applying the first law of thermodynamics to the system, the following expression is obtained:

$$
w_{\mathrm{N}}=-\left(q_{2 / 2 \mathrm{a}-3}+q_{5 / 5 \mathrm{a}-6}\right),
$$

where $q_{2 / 2 a-3}$ is the heat transferred from the system to the heat sink and $q_{5 / 5 a-6}$ is the heat transferred from the heat source to the system. Looking at this situation from a strictly thermodynamic point of view we can see from eqs. (1) and (2) that in the first two processes of the cycle the work is done on the system $\left(w_{1-2 / 2 a}\right.$ and $\left.w_{2 / 2 a-4}\right)$ and in the third process the system performs work ( $\left.w 4-5 / 5 a\right)$. However, in a real device, the work performed by the system in the third process represents some potential electric energy that could be recovered (we are actually recovering

the energy that was used to charge the ECM) during the process of depolarizing the ECM. A special electric circuit (see, for example, that presented in ref. [30]) should be used to recover this energy; however, since some irreversibilities in such an electric circuit are inevitable (or the circuit is not used at all), we will define a degree of the energy recovery $n$, which indicates what percentage of the available discharge electric energy is successfully recovered. The total work performed by the system can now be expressed as

$$
w_{\mathrm{N}, \mathrm{R}}=w_{1-2 / 2 \mathrm{a}}+w_{2 \mathrm{a} / 2-4}+\frac{n}{100} \times w_{4-5 / 5 \mathrm{a}} .
$$

By combining eq. (2), eq. (3) and eq. (4) we obtain

$$
w_{\mathrm{N}, \mathrm{R}}=-\left(q_{2 / 2 \mathrm{a}-3}+q_{5 / 5 \mathrm{a}-6}\right)-w_{4-5 / 5 \mathrm{a}} \times\left(1-\frac{n}{100}\right) .
$$

Next, we define the COP of the system as

$$
\mathrm{COP}=\frac{q_{5 / 5 \mathrm{a}-6}}{w_{\mathrm{N}, \mathrm{R}}} .
$$

In the literature dealing with the energy efficiency of electrocaloric-cooling devices [22-24,27,31], the net work was up to date calculated as the sum of the heat crossing the boundary of the system under investigation. From eq. (5) it can be seen that the net work performed on the systems equals the sum of the heat crossing its boundary only when the degree of the electric-energy recovery equals $100 \%$. However, it is not clear whether the authors of the studies were aware of the necessity for the electricenergy recovery. What can also be concluded is that the irreversibilities occurring during the process of electricenergy recovery have not been considered until now when estimating the energy efficiency of electrocaloric-cooling devices.

Electrocaloric properties of PMN-100xPT. - We focus on a system employing PMN-100xPT bulk ceramics with $x=0, x=0.1$, and $x=0.35$. For reasons of simplicity it is assumed that the specific heat $\left(c_{E}\right)$, the dipolar entropy change $\left(\Delta s_{P}\right)$ and the entropy generation due to the hysteresis $\left(\Delta s_{H}\right)$ of the ECMs are constant in the temperature range in which the system operates. Since we define the system to operate around room temperature and, for the selected ECMs the phase transition due to the temperature change or the applied electric field is not expected around room temperature [32-35], this is a good approximation. The dipolar entropy change of ECM can be expressed via the adiabatic temperature change of the ECM [36] and can be written as 


$$
\Delta s_{\mathrm{P}}=c_{E} \times \ln \left(\frac{T}{T-\Delta T_{\mathrm{EC}, \mathrm{M}}}\right)+\Delta s_{\mathrm{H}},
$$

where $C E$ is the specific-heat capacity at zero electric field and $300 \mathrm{~K}$ and $\Delta T_{E C, M}$ is the measured adiabatic temperature change of the PMN-100xPT at $300 \mathrm{~K}$ and at $\Delta \mathrm{E} \approx 5.5 \mathrm{MV} / \mathrm{m}$. The specific-heat capacity of the PMN-100xPT was measured using a differential scanning calorimeter DSC (Netzsch, DSC $204 \mathrm{~F} 1$ ). The $\triangle T_{E C, M}$ of PMN and PMN-10PT were obtained from the literature [13,29]. On the other hand, to obtain the $\Delta \mathrm{T}_{\mathrm{EC}, \mathrm{M}}$ of the PMN-35PT, direct measurements were preformed. The results of the measurements are presented in the next section.

To calculate $\Delta \mathrm{s}_{\mathrm{H}}$, polarization-electric field curves were measured under conditions corresponding to the process $1-2 / 2 a$ and the process $4-5 / 5 a$. The results of the measurements can be seen in fig. 3 and $\Delta \mathrm{s}_{H}$ is calculated as described in ref. [37]:

$$
\Delta s_{\mathrm{H}}=\int \frac{P_{\mathrm{IRR}} \times \mathrm{d} E}{2 T},
$$

where PIRR is the irreversible polarization function. Now, the adiabatic temperature change of the ECM at any initial temperature $T$ can be estimated as

$$
\Delta T_{\mathrm{EC}}=T \times\left[\exp \left(\frac{\Delta s_{\mathrm{H}}-\Delta s_{\mathrm{P}}}{c_{E}}\right)-1\right] .
$$

Preparation and electrocaloric measurements of PMN-35PT. - For the synthesis of the PMN-35PT ceramic powder, $\mathrm{PbO}$ (99.9\%, Aldrich), MgO (98\%, Aldrich), TiO2 (99.8\%, Alfa Aesar) and Nb2O5 (99.9\%, Aldrich) were mixed in the stoichiometricmolar ratio. The homogenized powder mixture was high-energy-milled in aplanetary mill (Retsch, PM 400) at $300 \mathrm{rpm}$ for 72 and additionally in an attritor mill (Netzsch, PE 075/PR 01) at $800 \mathrm{rpm}$ for $4 \mathrm{~h}$ in isopropanol.

The powder was analysed by X-ray diffractometer (XRD, X'Pert PRO MPD, PANalytical, Almeo) using $\mathrm{CU}-\mathrm{K} 1$ radiation. The XRD patterns were recorded in the 2-region from $10^{\circ}$ to $70^{\circ}$ using the $1 \mathrm{D}$ detector (XCelerator, PANalytical, Almeo) with the capture angle of 2.122 $2^{\circ}$.The exposure time for each step was $100 \mathrm{~s}$ and the interval between the obtained data points was $0.034^{\circ}$. The XRD pattern of the as-synthesized powder corresponded to the perovskite phase only (JCPDS 81-0861).

The powder compacts were pressed uniaxially at $50 \mathrm{MPa}$ and then isostatically at $300 \mathrm{MPa}$ and sintered in double alumina crucibles in the presence of the PMN-35PT packing powder at 1473K (1200 ${ }^{\circ} \mathrm{C}$ ) for $2 \mathrm{~h}$. Heating and cooling rates were $2 \mathrm{~K} / \mathrm{min}$. The XRD patterns of the crushed pellets were recorded under the same conditions as described above and confirmed the perovskite phase only (JCPDS 81-0861). The relative density of the sintered pellets, measured with Archimedes method, of the sample was $96.4 \%$. The average grain size, determined from the scanning electron microscope images (JEOL JSM- 7600) of the thermally etched samples, was $(1.1 \pm 0.5) \mathrm{m}$. For the polarizationelectric field (P-E) and electrocaloric measurements, the ceramic pellets were cut and polishedto $200 \mathrm{~m}$ and $80 \mathrm{~m}$, respectively. Afterwards, the samples were heated at $873 \mathrm{~K}\left(600{ }^{\circ} \mathrm{C}\right)$ for $1 \mathrm{~h}$, and cooled with the rate of $1 \mathrm{~K} / \mathrm{min}$. The $\mathrm{Cr} / \mathrm{Au}$ electrodes were deposited on the faces by RF-magnetron sputtering (5 pascal).

Electrocaloric properties of the PMN-35PT were studied by using a high-resolution calorimeter, which allows precise temperature stabilization (within $0.1 \mathrm{mK}$ ) and high-resolution measurements of the sample temperature. The details of the measurement procedure can be found in ref. [13]. The electrocaloric temperature change $\Delta T_{E C, M}$, measured at different electric-field amplitudes as a function of temperature, of the PMN-35PT bulk ceramics is shown in fig. 4.

In the vicinity of the room temperature, i.e. at $300 \mathrm{~K}$, the $\Delta \mathrm{T}_{\mathrm{EC}, \mathrm{M}}$ was $0.23 \mathrm{~K}$ and $0.51 \mathrm{~K}$ at 28 $\mathrm{kV} / \mathrm{cm}$ and $60 \mathrm{kV} / \mathrm{cm}$, respectively. With increasing temperature the $\Delta \mathrm{T}_{\mathrm{EC}, \mathrm{M}}$ increased and it reached $0.94 \mathrm{~K}$ at $60 \mathrm{kV} / \mathrm{cm}$ and $380 \mathrm{~K}$. During the direct EC measurements no Joule heating of the ceramics was observed. 


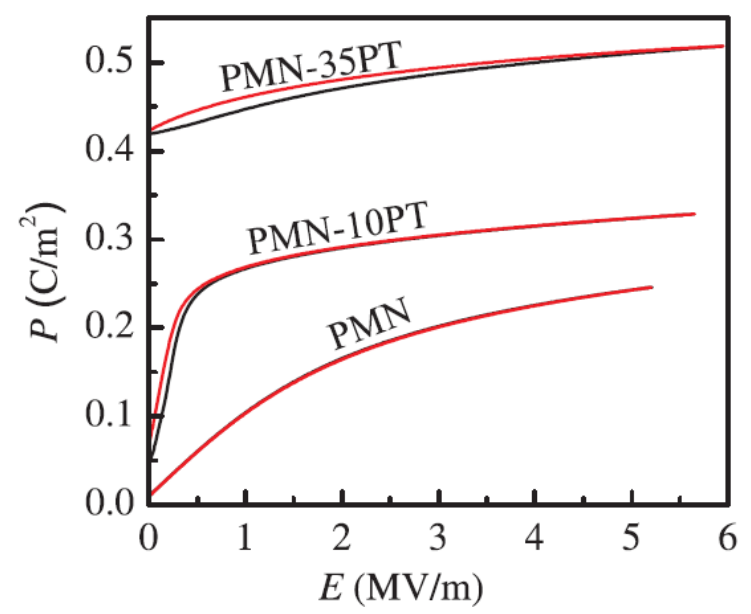

Fig. 3: (Colour on-line) Polarization-electric field curves for the PMN-100xPT with $x=0,0.1$ and 0.35 at $300 \mathrm{~K}$ and at $\Delta \mathrm{E} \approx 5.5 \mathrm{MV} / \mathrm{m}$. The P-E measurements of the PMN-100xPT were performed at $300 \mathrm{~K}$ using an Aixacct TF analyser 2000. A triangular electric signal with rise and fall times of $50 \mathrm{~ms}$ was used. Ten or more cycles were applied to the sample so that repeatable $P(E)$ results were obtained.

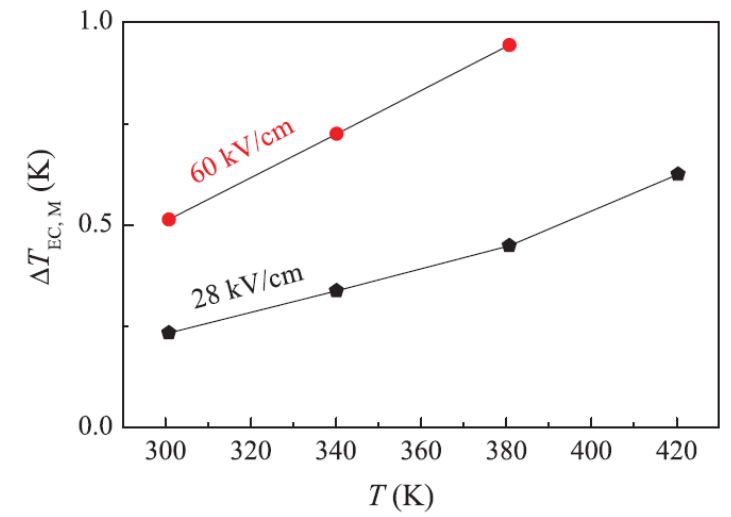

Fig. 4: (Colour on-line) The $\Delta T_{E C, M}$ of the PMN-35PT vs. temperature, measured at $28 \mathrm{kV} / \mathrm{cm}$ and 60 $\mathrm{kV} / \mathrm{cm}$.

Energy efficiency of the system. - First, the heat crossing the boundary of the system is defined as

$$
\begin{aligned}
& q_{2 / 2 \mathrm{a}-3}=\left(T_{3}-T_{2 / 2 \mathrm{a}}\right) \times c_{E}, \\
& q_{5 / 5 \mathrm{a}-6}=\left(T_{6}-T_{5 / 5 \mathrm{a}}\right) \times c_{E} .
\end{aligned}
$$

Furthermore, using the data presented in fig. 3 the work w4-5/5a is calculated as

$$
w_{4-5 / 5 \mathrm{a}}=\int_{E_{2}}^{E_{1}} E \times \mathrm{d} D .
$$

Now we define the ratio $\xi$ (also known as the exergy efficiency) as

$$
\xi=\left(\mathrm{COP} / \mathrm{COP}_{\mathrm{C}}\right) \times 100,
$$

where COP is the coefficient of performance of the system under investigation, calculated according to eq. (5) and COPC is the coefficient of performance of the Carnot heat pump, working between the average temperatures at which the heat is transferred from our system to the heat source and the heat sink, respectively.

The results of the analysis are presented in fig. 5 where the ratio $\xi$ is presented as a function of $\mathrm{n}$. The analysis was performed for four different temperature spans (no heat regeneration (OK), $5 \mathrm{~K}$, $15 \mathrm{~K}$ and $30 \mathrm{~K}$ ) and in all the cases the temperature of the heat sink was defined at 300K. Furthermore, 
to investigate the influence of the hysteresis on the efficiency of the system, the analyses were carried out by neglecting the hysteresis $\left(\Delta \mathrm{s}_{\mathrm{H}}=0\right)$ and by taking the hysteresis into account.

Let us first focus on the results of the analyses neglecting the hysteresis. If no heat is regenerated and no electric energy is recovered, $\xi$ does not exceed $5 \%$, regardless of the selected PMN-100xPT. By recovering the electric energy, the efficiency can be substantially increased; however, it needs to be pointed out that to achieve a high $\xi$ a large part of the electric energy should be recovered. Even when $\mathrm{n}$ is $95 \%, \xi=40 \%$ for PMN, $\xi=33 \%$ for PMN-10PT and $\xi=15 \%$ for PMN35PT; however, $\xi$ approaches $100 \%$ when $\mathrm{n}$ is increased to $100 \%$.

When the heat regeneration is also taken into consideration, the is much less dependent on the $\mathrm{n}$. In the case in which $\Delta \mathrm{Tspan}=30 \mathrm{~K} \xi=48 \%$ for PMN, $\xi=50 \%$ for PMN-10PT and $\xi=38 \%$ for PMN-35PT even if no electric energy is recovered.

Looking at the heat regeneration and the electric-energy recovery combined, we can see from fig. 5 that a high $\xi$ can be reached. For example, if $\Delta$ Tspan $=30 \mathrm{~K}$ and a realistic value of $n=80 \%[10,30]$ is considered, $\xi=81 \%$ for PMN, $\xi=82 \%$ for PMN-10PT and $\xi=74 \%$ for PMN-35PT.

When the hysteresis of the material is accounted for as well, we can see that it has almost no effect on the $\xi$ for the PMN. This was expected since the hysteresis loop of the relaxor PMN is very slim at 300K. On the other hand, when the PMN-10PT and PMN-35PT are considered, we can see that the hysteresis can cause a relatively large drop in the efficiency, especially for cases with high-n numbers and low-temperature spans.

(a)

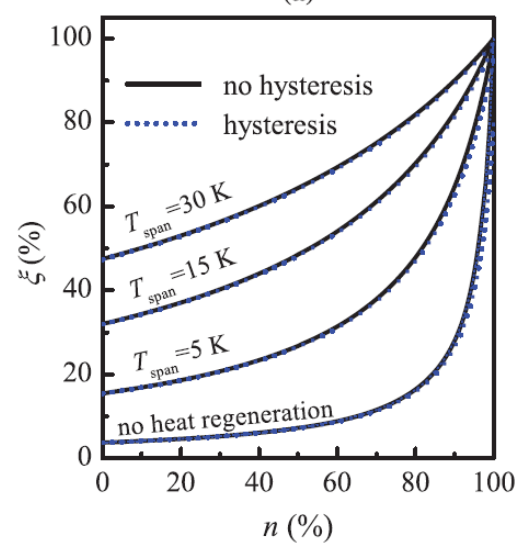

(b)

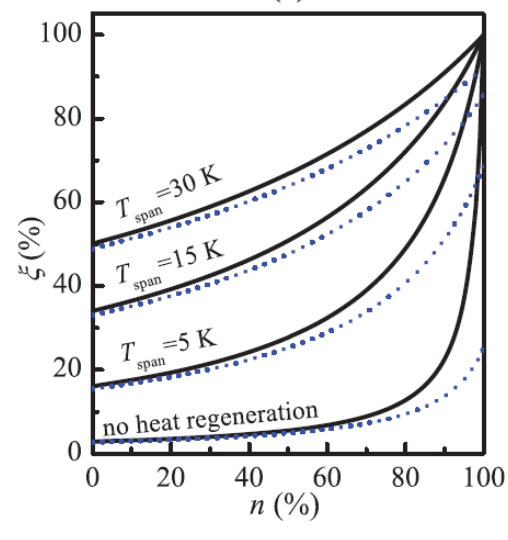

(c)

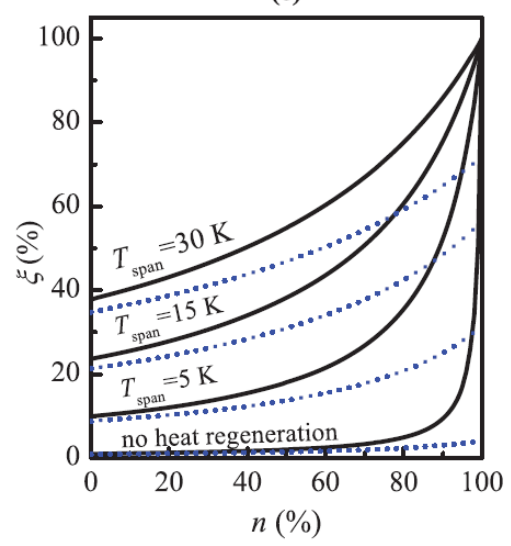

Fig. 5: (Colour on-line) The exergy efficiency of the system under investigation as a function of the degree of electric-energy recovery $n$ for (a) PMN, (b) PMN-10PT and (c) PMN-35PT.

Conclusion. - The above-presented results show the importance of electric-energy recovery and heat regeneration on the achievable energy efficiency of an electrocaloric cooling device employing PMN$100 x$ PT ceramics. It can be concluded that a high degree of electric-energy recovery, preferably combined with a heat regeneration process, is needed to create a basis for an efficient electrocaloriccooling system. Furthermore, the selected ECM should possess no, or only a slim, electric hysteresis.

$$
* * *
$$

The authors wish to thank Brigita Rožič for her valuable remarks and comments. The authors also thank the Slovenian Research Agency for financial support in the frame of the projects PR-05025, L26768, J2-6779 and programs P2-0105 and P1-0125.

\section{REFERENCES}

[1] Ožbolt M., Kitanovski A., Tušek J. and Poredoš A., Int. J. Refrig., 40 (2014) 174.

[2] Correia T. and Zhang Q. (Editors), Electrocaloric Materials (Springer Verlag, Berlin, Heidelberg) 2014, p. 253. 
[3] Kitanovski A., Tušek J., Tomc U., Plaznik U., Ožbolt M. and Poredoš A., Magnetocaloric Energy Conversion - From Theory to Applications (Springer Verlag, Berlin, Heidelberg) 2015, p. 456.

[4] Kutnjak Z., Rožič B. and Pirc R., Electrocaloric Effect: Theory, Measurements, and Applications, Wiley Encyclopedia of Electrical and Electronics Engineering (Wiley) 2015, pp. 1-19.

[5] Kobeko P. and Kurtschatov J., Z. Phys., 66 (1930) 192.

[6] Wiseman G. G. and Kuebler J. K., J. Phys. Rev., 131 (1963) 2023.

[7] Scott J. F., Annu. Rev. Mater. Res., 41 (2011) 229.

[8] Moya X., Kar-Narayan S. and Mathur N. D., Nat. Mater., 13 (2014) 439.

[9] Alpay S. P., Mantese J., Trolier-McKinstry S., Zhang Q. and Whatmore R. W., MRS Bull., 39 (2014) 1099.

[10] Moya X., Defay E., Heine V. and Mathur N. D., Nat. Phys., 11 (2015) 202.

[11] Sebald G., Pruvost S., Seveyrat L., Lebrun L., Guyomar D. and Guiffard B., J. Eur. Ceram. Soc., 27 (2007) 4021.

[12] Valant M., Dunne L. J., Axelsson A.-K., Alford N. M., Manos G., Peräntie J., Hagberg J., Jantunen H. and Dabkowski A., Phys. Rev. B, 81 (2010) 214110.

[13] Rožič B., Malič B., Uršič H., Holc J., Kosec M. and Kutnjak Z., Ferroelectrics, 421 (2011) 103.

[14] Peräntie J., Tailor H. N., Hagberg J., Jantunen H. and Ye Z.-G., J. Appl. Phys., 114 (2013) 174105.

[15] Mischenko A. S., Zhang Q., Scott J. F., Whatmore R. W. and Mathur N. D., Science, 311 (2006) 1270.

[16] Scott J. F., Science, 315 (2007) 954.

[17] Lu S. G., Rožič B., Zhang Q. M., Kutnjak Z., Li X., Pirc R., Gorny L. J. and Lin M., Appl. Phys. Lett., 97 (2010) 202901.

[18] Peng B., Fan H. and Zhang Q., Adv. Funct. Mater., 23 (2013) 2987.

[19] Neese B., Chu B., Lu S. G., Wang Y., Furman E. and Zhang Q. M., Science, 321 (2008) 821.

[20] Lu S. G., Rožič B., Zhang Q. M., Kutnjak Z., Li X., Furman E., Gorny L. J., Lin M., Malič B., Kosec M., Blinc R. and Pirc R., Appl. Phys. Lett., 97 (2010) 162904.

[21] Zhang G., Li Q., Gu H., Jiang S., Han K., Gadinski M. R., Haque M. A., Zhang Q. and Wang Q., Adv. Mater., 27 (2015) 1450.

[22] Gu H., Craven B., Qian X., Li X., Cheng A. and Zhang Q. M., Appl. Phys. Lett., 102 (2013) 112901.

[23] Gu H., Qian X. S., Ye H. J. and Zhang Q. M., Appl. Phys. Lett., 105 (2014) 162905.

[24] Guo D., Gao J., Yu Y. J., Santhanam S., Slippey A., Fedder G. K., McGaughey A. J. H. and Yao S. C., Int. J. Heat Mass Transfer, 72 (2014) 559.

[25] Sinyavsky Y. V. and Brodyansky V. M., Ferro- electrics, 131 (1992) 321.

[26] Karmanenko S. F., Pakhomov O. V., Prudan A. M., Starkov A. S. and Eskov A., J. Eur. Ceram. Soc., 27 (2007) 3109.

[27] Epstein R. I. and Malloy K. J., J. Appl. Phys., 106 (2009) 064509.

[28] Gu H., Qian X., Li X., Craven B., Zhu W., Cheng A., Yao S. C. and Zhang Q. M., Appl. Phys. Lett., 102 (2013) 122904.

[29] Plaznik U., Kitanovski A., Rožič B., Malič B., Uršič H., Drnovšek S., Cilenšek J., Vrabelj M., Poredoš A. and Kutnjak Z., Appl. Phys. Lett., 106 (2015) 043903.

[30] Campolo D., Sitti M. and Fearing R. S., IEEE Trans. Ultrason. Eng., 50 (2003) 237.

[31] Ožbolt M., Kitanovski A., Tušek J. and Poredoš A., Int. J. Refrig., 37 (2014) 16.

[32] Kutnjak Z., Vodopivec B. and Blinc R., Phys. Rev. B, 77 (2008) 054102.

[33] Eugene V. C., Nikolai K. Y. and Dwight V., Appl. Phys., 83 (1998) 3298.

[34] Kelly J., Leonard M., Tantigate C. and Safari A., J. Am. Ceram. Soc., 80 (1997) 957.

[35] Trefalt G., Malič B., Holc J., Uršič H. and Kosec M., J. Am. Ceram. Soc., 95 (2012) 1858.

[36] Pirc R., Kutnjak Z. and Blinc R., Appl. Phys. Lett., 98 (2011) 021909.

[37] Brey W., Nellis G. and Klein S., Int. J. Refrig., 47 (2014) 85. 